\title{
Microbial and immunological investigations and remedial action after an outbreak of humidifier fever
}

\author{
J H EDWARDS
}

From the Medical Research Council Pneumoconiosis Unit, Llandough Hospital, Penarth, S Glam, UK

ABSTRACT Humidifier fever (Monday sickness) occurring in office staff in a factory processing rayon presented as pyrexia with a polyuria and leucocytosis on the first day back to work after a break during the winter half of the year. Chest radiographs showed no abnormalities but pulmonary function tests indicated mild airways obstruction in the affected group as a whole. Respirable dust samples taken on a Monday when 11 cases occurred were not pyrogenic, indicating that a mechanism other than direct pyrogen activity produced the pyrexia. Efforts were then directed to determining an immunological basis for the episodes. In particular, Thermoactinomyces vulgaris, previously held responsible for humidifier fever, was studied. During the episode of 11 cases, the number of viable airborne spores of this organism was far higher than on Mondays when no cases occurred. In a second episode of nine cases, however, the airborne viable count was of the same order as nonepisode Mondays.

Extracts of $T$ vulgaris produced lines of precipitation in gel diffusion studies with roughly half the office staff sera tested, but no correlation was observed between precipitin line formation and disease. A similar proportion of normal sera reacted against this extract. Extracts of dust lying on the topside surface of the suspended ceiling above the office, however, produced precipitin lines with sera from $16 / 18$ affected individuals and $2 / 18$ non-affected individuals $(p<0.001)$ as did extracts of humidifier material.

Extensive microbial analysis failed to detect any one fungus or bacterium that produced antigens capable of reacting with positive serum, but extracts of amoebae correlated absolutely with humidifier material and ceiling dust extract in gel diffusion studies. A reaction of identity observed between the amoebae and ceiling dust extracts showed the presence of identical antigens. In similar studies the high degree of cross reactivity with antigens and sera from Spanish and Swedish outbreaks was obtained, which suggested a common antigen source in humidifier fever.

That these antigens were produced by microbial development on rayon fibre could be shown by incubating rayon dust from the factory atmosphere with sterile water and testing with sera from affected individuals. Bales of rayon entering the factory did not have this potential to develop antigens, indicating microbial contamination after handling and processing. The initial source of contamination was considered to be the humidifier disseminating microbial spores and cysts throughout the factory and on to the suspended ceiling above the office. These were capable of secondary development on settled rayon fly under wet conditions, and evidence for this was obtained. Remedial action included cleaning the humidifier, modifying the baffle plates, running water to waste, and installing a prefilter. Dust was eliminated from the office area, and new accommodation, including the building of an office block detached from the main factory, was arranged for the office workers. So far no further cases have been reported.

In environments where people are present for any length of time temperature and humidity control is

Received November 1978 Accepted 29 March 1979 necessary to maintain comfortable working conditions. Similarly, certain work processes require fairly strict control of these variables, and this is achieved in several ways. In one of the more common methods $^{1}$ air is drawn in from the factory en- 
vironment and mixed with a variable proportion of external air. This is warmed and passed through a humidifier chamber where fine droplets of water are sprayed into the moving air current. Larger droplets are removed by baffle plates that cause turbulence and particle deposition. The temperature-controlled, humidified air is then distributed.

Normally these systems present few health problems except when infective or allergenic materials find their way into the humidification system. This can occur when organic material present either in the air taken into the humidifier, or in the water used for humidification, provides nutrient for air- or water-borne microbes. Biomass build-up occurs in the humidifier chamber itself or on the baffle plates due to air/water-borne particle deposition and may be propagated if the water that has not evaporated in the humidifier is recirculated. One of the first indications of an allergic response due to humidification was provided by Pestalozzi in $1959^{2}$ and since then numerous reports have appeared ${ }^{34}$ Although, as might be expected from the open nature of such systems, microbial analysis has shown the presence of many species of organisms in humidifier water, ${ }^{15}$ by analogy with other hypersensitivity lung diseases one might expect one organism to be the predominant sensitiser. In 1970 Banaszak et $\mathrm{al}^{3}$ reported that Thermoactinomyces vulgaris was the organism responsible for antigenic stimulus in humidifier fever since the organism had been isolated from an air conditioning system, was associated with other forms of hypersensitivity lung disease, and produced lines of precipitation on gel diffusion testing with sera from cases of humidifier fever. Inhalation challenge with extracts of $T$ vulgaris produced pyrexia in a case of humidifier fever. Our experience, however, is that extracts of $T$ vulgaris can react non-specifically on gel diffusion and the organism can activate the alternative pathway of complement. ${ }^{6}$ This latter property may have caused the induced pyrexia reported by Banaszak et al. ${ }^{3}$

An opportunity to study the role of $T$ vulgaris and other organisms in humidifier fever arose when an outbreak of the disease occurred in a local factory processing rayon into non-woven fabrics. Our investigations were undertaken to correlate airborne $T$ vulgaris organisms with disease incidence and also to determine the presence of other organisms that may have been active either non-specifically by pyrogen activity or specifically by immunological mechanisms. Since rayon was being processed continuously at the factory it had to be determined whether incoming bales were a source of contamination or whether this arose during handling and processing. Also, with material and sera available from two other outbreaks of humidifier fever in Spain and Sweden, it was possible to compare sources from geographically unrelated areas for similar characteristics. Finally, during the course of this study, remedial action was taken, and this is presented.

\section{Materials and methods}

DISEASE OCCURRENCE AND DISTRIBUTION The disease manifested itself as a pyrexial episode occurring on Mondays during the winter months or on the first day back to work after a holiday. The individuals affected were, in total, 20 of 50 office workers, but the number responding at any one time varied, and episodes were not seen every Monday. The other features of the disease were polyuria and leucocytosis. There were no abnormal radiograph findings, presumably reflecting a milder form of the disease compared with other studies. ${ }^{34}$ Lung function studies $\left[\mathrm{VA}^{1}=\right.$ alveolar volume (allowing for mixing); $T_{L}{ }^{1}=$ transfer factor (for carbon monoxide); $\mathrm{KCO}=\mathrm{TL}_{\mathrm{L}}{ }^{1} / \mathrm{VA}^{1}$ (transfer per unit volume); PFR = peak flow rate; $\mathrm{FEV}_{1}=$ forced expiratory volume (at $1 \mathrm{sec}) ; \mathrm{FVC}=$ forced vital capacity], performed on a Monday when 11 cases were recorded, showed evidence of a mild airways obstruction in the group of individuals most often affected. Both men and women were affected with an even spatial distribution within the office. There was no correlation between nonsmoking and increased serological activity as has been seen in farmer's lung, ${ }^{7}$ neither was the presence of rheumatoid factor detected (cf Banaszak et $a l^{3}$ ). A more detailed account of these findings is to be presented elsewhere.

\section{DESCRIPTION OF WORK AREA}

The factory processed rayon, and the main humidifier, although located above the main office, extracted and delivered air only out of and into the factory, which was separated from the offices by a fire-brick wall. A scale diagram of the main office area is shown in fig 1. Some gaseous interchange took place through holes in the wall and doors but the main pressure equilibration vents between the factory and the offices had been closed a few years before the investigation. An integral part of the scheme was a suspended ceiling over the office area producing a void space $117 \times 21.3 \mathrm{~m}$ in area and averaging $5.7 \mathrm{~m}$ to the roof. During the period when the pressure equilibration vents were in operation and due to inadvertent gaseous interchange-that is, doors open-rayon dust (fly) from the factory, as well as materials associated with building-for example, calcium sulphate and other debris-had 


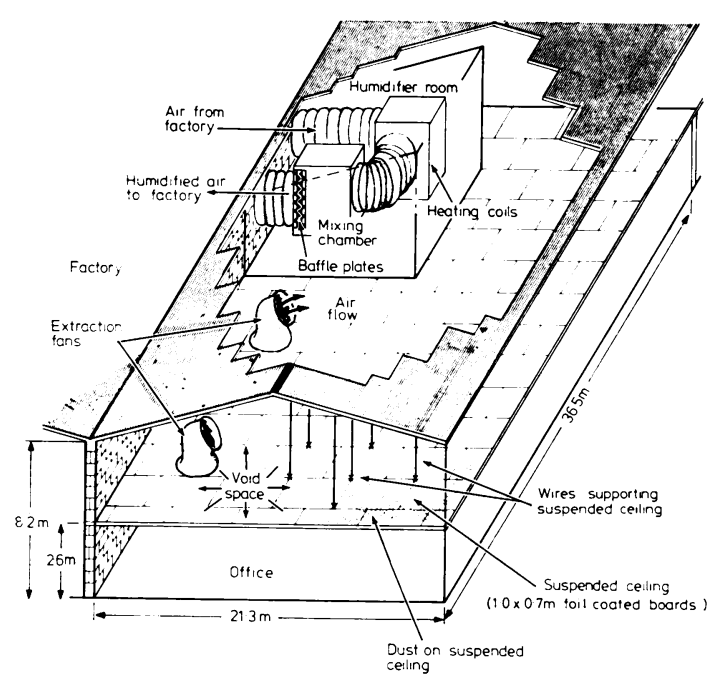

Fig 1 Diagram of main office area showing suspended ceiling and void space. Walls within the office area are not included. Air conditioning of office was provided by three extraction fans. A larger extraction fan was housed in roof distant to offices. Fresh air was obtained only by opening windows and doors. A subsidiary office, $30 \mathrm{~m}$ from main office, also had an extraction fan operating

thereby drawing dust in from void space.

settled on the top surface of the suspended ceiling.

The air conditioning for the offices merely extracted air from the office area into the void above. Equilibration took place through window/ door leaks but mainly through the unsealed joints between the $1.0 \times 0.7 \mathrm{~m}$ foil-coated boards that formed the suspended ceiling. This could be observed directly using the flame direction of a lighted match and by the presence of dust streaks down walls under the joints.

\section{AIR SAMPLING IN OFFICE AREA}

\section{Airborne microbes}

For 14 consecutive days air was sampled by an Andersen sampler ${ }^{8}$ at two points, one in the main office and the other in a minor office where two of the most frequently affected individuals worked. Air was drawn over nutrient agar plates at $28.3 \mathrm{l} / \mathrm{min}$ for five minutes, and samples were incubated at $50^{\circ} \mathrm{C}$ for two days to detect thermophiles. This was repeated with Sabouraud agar plates incubated at $37^{\circ} \mathrm{C}$ to detect fungi. After the two-week period air was sampled on three successive Mondays.

\section{Respirable dust}

Respirable fractions of dust were collected daily over the two-week period on $0.8 \mu \mathrm{m}$ Millipore filters and assayed gravimetrically. A comparison was made with the external environment.

\section{Detection of pyrogenic material in office dust and} microbial extracts

Dust from the Millipore membrane retaining the respirable fraction particles, collected on the Monday when 11 cases of humidifier fever were recorded, was used. It was resuspended in saline such that $1 \mathrm{ml}$ contained the amount of dust calculated to be inhaled by a sedentary worker over a work period of eight hours ${ }^{9}$ scaled down in the ratio 70 to 2.5 (the weight of an average man $(70 \mathrm{~kg})$ compared with the average weight of the rabbits $(2.5 \mathrm{~kg})$ used in the pyrogen study).

One $\mathrm{ml}$ of dust suspension was inoculated into the marginal ear veins of two rabbits and temperatures were recorded by indwelling rectal thermometers over a seven-hour period. The experiment was repeated with the dust suspension at $\times 2, \times 5$, and $\times 10$ this concentration. The ceiling dust itself was tested for pyrogenicity at $400 \mu \mathrm{g} / \mathrm{kg}$, likewise extracts of the organisms associated with the ceiling dust and office atmosphere-that is, $T$ vulgaris, Cladosporium herbarum, and a bacillus.

INVESTIGATIONS INTO CEILING DUST AND HUMIDIFIER MATERIAL

\section{Microbial analysis}

Fungi were grown on Sabouraud's agar and corn meal agar at room temperature and $37^{\circ} \mathrm{C}$. Bacteria were cultured on nutrient agar at room temperature, $37^{\circ} \mathrm{C}$, and $50^{\circ} \mathrm{C}$. Protozoa were detected by growth on Klebsiella aerogenes streaked on non-nutrient agar plates at room temperature.

\section{Preparation of extracts}

Dust extracts-The suspended ceiling tiles had a thin aluminium covering on their uppermost surface, and it was possible to reach several of these. The dust was mopped off these tiles with a wet sponge and extruded into a plastic bucket containing sterile deionised water. After several tiles had been cleaned the dust suspension was shaken and soluble material obtained by filtration. Concentration was by air dialysis. Typically, $200 \mathrm{~g}$ dust yielded $50 \mathrm{mg}$ soluble extract. To unprocessed suspension, phenol was added to $1 \%$ to prevent microbial development.

Humidifier material-The sludge from the humidifier chamber or baffle plates was extracted with water at $10 \% \mathrm{w} / \mathrm{v}$, filtered, and concentrated. Humidifier recirculating water was filtered and concentrated. 
Microbial extracts-Fungal extracts were prepared by growing isolated fungi in casein hydrolysate medium at room temperature or at $37^{\circ} \mathrm{C}$. Organisms were filtered off and the filtrate was dialysed and concentrated. The organisms were extracted by decompression rupture in a Hughes press. Bacterial extracts were prepared by double dialysis ${ }^{10}$ and cells extracted by a Hughes press.

Protozoal extracts-Gram-negative organisms (mainly $K$ aerogenes) were spread uniformly on a non-nutrient agar plate or corn meal agar plate and protozoa - that is, ciliates or amoebae - were seeded in a single streak. After development at room temperature (about 10 days) the agar was extracted with $1 \%$ phenol, concentrated, and dialysed.

\section{Detection of antigenic material in dust and microbial extracts}

The agar double gel diffusion technique was used to detect precipitating antibody in serum and antigenic material in extracts.

Serum was run into $10 \mathrm{~mm}$ diameter central wells cut in $1 \%$ agar in McIlvaine's citrate-phosphate buffer, pH 7.2, and extracts were run into $4 \mathrm{~mm}$ diameter peripheral wells in hexagonal arrangement $10 \mathrm{~mm}$ centre to centre apart.

Plates were incubated at room temperature for two days and stained according to techniques previously outlined. ${ }^{11}$

Precipitin lines from adjacent antigen wells that fuse at their intercept indicate the same antigenic material to be present in both wells (line of identity).

\section{Contamination of processed rayon dust obtained \\ from the atmosphere and incoming rayon}

Ten $\mathbf{g}$ aliquots of sterile (autoclaved) or untreated airborne rayon fly from the dust filtration units in the factory were incubated with $500 \mathrm{ml}$ aliquots of sterile (autoclaved) or untreated incoming factory water for 14 days at room temperature in sterile one litre conical flasks; $100 \mathrm{ml}$ were then removed, filtered, and concentrated to about $0.5 \mathrm{ml}$ by air dialysis.

One sample of untreated dust plus untreated water was processed after overnight incubation at $4^{\circ} \mathrm{C}$, and $100 \mathrm{ml}$ of untreated incoming water were also filtered and concentrated to $0.5 \mathrm{ml}$. The procedure was duplicated in its entirety using laboratory water in the place of factory water.

Thirty-six further samples of rayon from the centres of unused incoming rayon bales were processed as described with $10 \mathrm{~g}$ rayon plus $500 \mathrm{ml}$ sterile laboratory water in sterile one litre conical flasks. The presence of antigen was detected using immunodiffusion against known positive serum from a humidifier fever case.
Comparisons of antigenic material and sera from other outbreaks

Extracts of humidifier sludge from two other outbreaks as well as sera from these outbreaks were tested by immunodiffusion using joining lines of identity to detect identical antigens and functional antibodies. One outbreak was in a Spanish factory processing nylon, the other occurred in Sweden.

\section{Results}

SEQUENTIAL MICROBIAL AND DUST ANALYSIS IN OFFICE AREAS

Figure 2 shows the airborne $T$ vulgaris colony forming units during the initial two-week study and on the subsequent three Mondays. The initial correlation between airborne $T$ vulgaris counts and disease production-that is, 11 cases on the first Monday-was not observed when colony counts approached zero on the last Monday when nine cases occurred. Airborne fungal counts were mainly $C$ herbarum and tended to follow $T$ vulgaris counts, although the merging of colonies made an accurate numerical assessment difficult.

Extracts of $\boldsymbol{T}$ vulgaris isolated produced one blur line of precipitation on gel diffusion with about $50 \%$ of sera from the office workers, but there was no correlation between precipitation reaction and

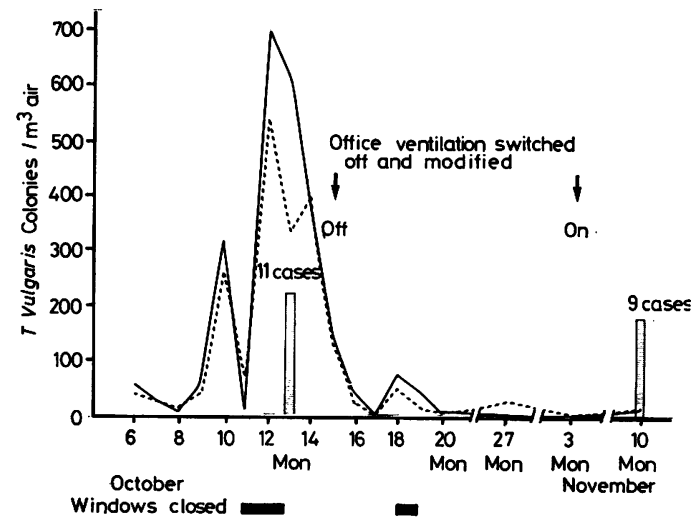

Fig 2 Number of Thermoactinomyces vulgaris colonies per cubic metre air taken in the main office - and subsidiary office - - - over a two-week period when episodes of humidifier fever occurred, and also on three successive Mondays afterwards. Note initial correlation between $T$ vulgaris counts and episodes on first Monday is not seen on fifth Monday with nine cases and low $T$ vulgaris counts. Modifications to ventilation system are shown as discussed in text. 
disease. The same extract produced the same percentage-positive reaction in normal sera tested, as was expected from our previous experience with this organism. ${ }^{6}$ Extracts of $C$ herbarum did not react on gel diffusion with sera tested.

There was no trend in the amount of respirable dust collected over the two-week period. Low levels of dust were observed from $0.082 \mathrm{mg} / \mathrm{m}^{3}$ to $0.204 \mathrm{mg} / \mathrm{m}^{3}$ in the general office (average 0.174 ) and $0.084 \mathrm{mg} / \mathrm{m}^{3}$ to $0.247 \mathrm{mg} / \mathrm{m}^{3}$ (average 0.15 ) in the smaller office. Outside air averaged $0.063 \mathrm{mg} / \mathrm{m}^{3}$. No correlation was seen between episodes of disease and airborne dust concentration.

\section{PYROGEN RESPONSES TO DUST AND}

ORGANISMS IN OFFICE ENVIRONMENT

Pyrogen was not detected in the office dust sample even with $\times 5$ and $\times 10$ relative amounts of dust (fig 3a). Ceiling dust (fig 3a) and calcium sulphate were also non-pyrogenic at up to $400 \mu \mathrm{g} / \mathrm{kg}$. Extracts of $T$ vulgaris and $C$ herbarum produced pyrexia (fig $3 \mathrm{~b}$ ). Interestingly, the response to $T$ vulgaris was biphasic at $400 \mu \mathrm{g} / \mathrm{kg}$ reducing to a late monophasic response at $40 \mu \mathrm{g} / \mathrm{kg}$. After these results $T$ vulgaris was considered unlikely to be the causative agent in our episodes.

\section{INVESTIGATIONS INTO CEILING DUST}

The investigation was extended into ceiling dust which, although non-pyrogenic, presumably con-



Fig 3a Pyrogen responses in rabbits to materials associated with a humidifier fever environment; ceiling dust $400 \mu \mathrm{g} / \mathrm{kg}$, respirable airborne dust $\times 1, \times 2, \times 5$, $\times 10$. Note absence of response to respirable airborne dust from office at up to $\times 10$ that inhaled by individuals, also the non-pyrogenic nature of ceiling dust at $400 \mu \mathrm{g} / \mathrm{kg}$.

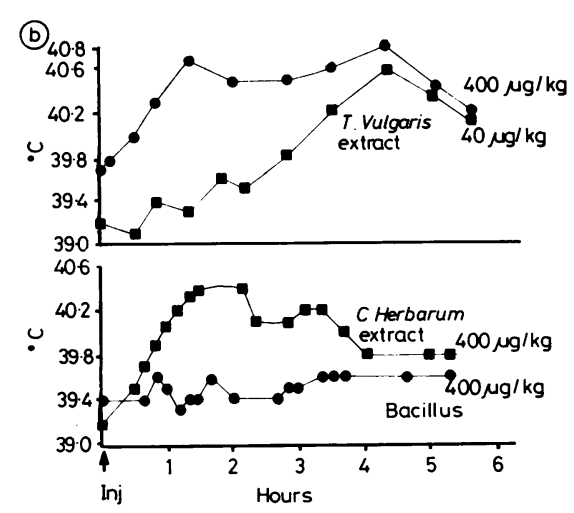

Fig 3b Pyrogen responses in rabbits to extracts of organisms associated with humidifier fever environment. Note biphasic pyrogen response to $T$ vulgaris extract at $400 \mu \mathrm{g} / \mathrm{kg}$, reducing to a late monophasic reaction when dose was $40 \mu \mathrm{g} / \mathrm{kg}$. C herbarum extract was also pyrogenic whereas an extract of Bacillus often found was not.

tained active material. An extract of this dust at $50 \mathrm{mg} / \mathrm{ml}$ produced lines of precipitation on gel diffusion with $16 / 18$ affected cases and 2/18 nonaffected sera. Statistically, this is significant $(\mathrm{p}<0.001)$. With many sera more than one line was observed.

The isolation of organisms produced many fungi and bacteria, some of which were identified-for instance, $C$ herbarum, Paecilomyces variotii, Mucor pusillus, Aspergillus fumigatus, $A$ niger, $T$ vulgaris, Bacillus subtilis; others were not identified but yeasts, bacilli and some Enterobacteriacae were detected. Extracts of all organisms were made regardless of identification. None of the organisms yielded extracts reacting as fully with positive sera as did an extract of the dust itself.

INVESTIGATIONS INTO HUMIDIFIER MATERIAL The inability to isolate organisms responsible for the antigens in the ceiling dust then led to investigations into the humidifier itself. The baffle plates and mixing chamber were thickly coated with wet rayon fly which formed a dark sludge with a light buff coloured drier surface. A metallic sheen was seen, which tended to float on water and adhere to glass surfaces. Extracts of this sludge and also concentrated recirculating water reacted even more strongly in gel diffusion than extracts of ceiling dust with positive sera. Microbial analysis showed different fungi and bacteria from the ceiling dustfor instance, Fusarium. Flavobacterium, Sphaerotilus. 
However, again no one extract was responsible for the antigens seen in the sludge extract. Microscopic analysis showed protozoa of two main groups, ciliates and amoebae. Purified ciliates did not produce an antigenic extract whereas amoebal extracts produced as described yielded up to five precipitation lines on gel diffusion with positive sera.

A known amoeba, Naegleria gruberi, grown on $K$ aerogenes or other Gram-negative organisms yielded antigens that produced lines of identity on gel diffusion with ceiling dust extract, ${ }^{12}$ and there was an absolute correlation between precipitation reactions to ceiling dust, sludge extract, and $N$ gruber $i$ extract, although the order of reactivity was sludge > ceiling dust $>$ amoebae. Certainly, amoeba extracts did not account for all the antigenic components in sludge.

The discovery of amoebae in the humidifier (provisionally identified as Enchinamoeba exundans) prompted an equivalent search in the ceiling dust and this also proved positive for amoeba belonging to the Vahlkampfiidae, provisionally Vahlkampfia sp.

POTENTIAL SOURCES OF ANTIGEN

DEVELOPMENT IN FACTORY DUST AND

INCOMING RAYON BALES

Overnight extracts of the factory rayon fly were negative antigenically as were 14-day extracts of autoclaved factory dust with sterile water. The addition of sterile water to the factory rayon fly led to antigen development (fig 4) but not the addition of factory or laboratory water to sterile fly. No antigens developed when incoming, that is, fresh rayon, was incubated with sterile water.

These experiments indicate that airborne factory dust can develop antigens and that "contamination" occurs only after the incoming rayon is processed. The spores and cysts may be attached to the rayon fly either during processing through machines (a dry process) or be picked up from the atmosphere when the fly becomes airborne. The source of these microbes could be the humidifier, since although the bulk (that is, $>99 \%$ ) material has been removed, the remaining sludge (about $100 \mathrm{~g}$ ) is still potently antigenic.

Further inquiries showed that on three distinct occasions the valve controlling the steam supply to a calorifier located over the office area with the humidification system stopped working. Steam filled the void space, and the entire suspended ceiling had pockets of water that sometimes caused the tiles to sag and leak into the office. This factor it seems led to secondary microbial development, since there was an appreciably increased presence of soluble sugars in the ceiling dust detected, using the



Fig 4 Antigen development in airborne factory dust, obtained from dust filtration units, incubated with factory or laboratory water for 14 days at room temperature (extracts $A, B, C, E, G, H, I)$. Extracts $D$ and $J$-water used concentrated $\times 200$. Extract $K-$ overnight extraction at $4^{\circ} \mathrm{C}$ of non-sterile dust with factory water. Extracts tested by immunodiffusion, development with positive serum.

A Dust plus factory water, B Dust plus sterile factory water, C Sterile dust plus factory water, D Factory water concentrated, E Sterile dust plus sterile factory water, $F$ Baffle plate sludge extract, $G$ Dust plus laboratory water, $H$ Dust plus sterile laboratory water, I Sterile dust plus laboratory water, J Laboratory water concentrated, and $K$ Dust, overnight extraction at $4^{\circ} \mathrm{C}$. Note development of antigens only in incubations of non-sterile dust and factory or laboratory waterthat is, $A, B, G$, and $H$ compared with $C$ and $I$. Factory and laboratory water supplies did not contain viable microbes capable of antigen production, $D$ and $J$. That development of micro-organisms was necessary is shown by extracts $E$ and $K$.

phenol/sulphuric acid method of Dubois et al, , $^{13}$ compared with factory rayon fly. To test whether the incoming rayon already had the necessary spores/ cysts for antigen development, 36 samples from the centre of incoming bales were incubated with sterile water and processed as for factory rayon fly samples. These results showed no antigens to be produced indicating that the factory rayon fly became contaminated from the humidifier air as it became airborne.

\section{ANTIGENIC COMPARISONS WITH MATERIALS}

FROM OTHER OUTBREAKS

Lines of identity formed between Spanish, Swedish, and our humidifier sludge extracts reacting with either local positive sera or with Spanish or Swedish sera. This suggests common antigen sources and types of antibody response in these three outbreaks. 


\section{REMEDIAL ACTION}

During the study period some attempts were made to provide a solution to the problem. After the initial 11-episode outbreak (fig 2) the fans extracting air from the office were switched off and a large fan was installed in the roof, removing air from the void. The office heating system was modified to take in external air and force this into the office. These actions sought to increase the air pressure in the office relative to the void space. Manometer readings, however, showed little difference, if any, between void and office, possibly due to the relatively large void volume $\left(12.6 \times 10^{3} \mathrm{~m}^{3}\right)$ compared with the office $\left(1.5 \times 10^{3} \mathrm{~m}^{3}\right)$. Also at this time the humidifier was cleaned. The next nine episodes (fig 2) called for more drastic remedial action, and the office workers were rehoused in temporary buildings while the problem was considered. The suspended ceiling was removed in its entirety $\left(2.5 \times 10^{3} \mathrm{~m}^{2}\right)$ and the area thoroughly wet cleaned. The carpet $\left(470 \mathrm{~m}^{2}\right)$ in the office area was removed as an extract of this showed antigen to be present. The fire-brick wall separating the factory and offices was sealed off. Finally, the humidifier (plus two others in the factory) was cleaned; $20 \mathrm{~kg}$ sludge was removed. The baffle plates were replaced by non-metallic plates that are easily removed and cleaned (now once a month). Most importantly, the intake air had a prefilter incorporated that removes about $99.9 \%$ of all the rayon fly. The water from the humidifier is run to waste and not recirculated. A recent examination of the humidifier showed less than $100 \mathrm{~g}$ of sludge in the whole system indicating fairly successful cleaning and preventative procedures. A new ceiling was placed on the office area, and half the workforce containing equal numbers of previously affected and non-affected individuals returned to the office area; so far ( 24 months) no episodes have been reported.

\section{Discussion}

The findings of our investigations suggest that in this outbreak at least the thermophilic actinomycete, $T$ vulgaris, did not play a causative role as had been suggested by Banaszak et $a .^{3}$ Possibly the precipitation lines seen in this American outbreak were due to non-specific reactions and the pyrexia observed on inhalation challenge was due to endogenous pyrogen from $T$ vulgaris. It is also unlikely that thermophiles can develop in a normal humidifier since the running temperature is too low $\left(<25^{\circ} \mathrm{C}\right)$. Again, farmers handling mouldy hay can be exposed to vast numbers of $T$ vulgaris spores ${ }^{14}$; yet our experience is that of more than a thousand sera tested over the past decade, only one farmer had precipitins to $T$ vulgaris over and above the blur line that occurs with about half normal sera. The source of antigen described here is partly or wholly protozoal but the laboratory development of the total antigenic complex has not as yet been achieved, possibly due to our inability to recreate the precise conditions prevailing in the humidifier. From the experiments performed the following sequence of events probably led to the episodes observed.

The airborne rayon fly became deposited in the humidifier. Air/water-borne fungi and bacteria developed on the rayon (modified cellulose). Concurrently, protozoa developed on the fungi/ bacteria setting up a sewage fungus complex. ${ }^{15}$ The products and spores or cysts of these organisms were blown into the factory and adhered to factory rayon fly. This passed initially through the equilibration vents between the factory and the void and settled on the suspended ceiling. Steam saturation provided the necessary moisture for setting up a secondary complex, which when dried produced the very fine antigenic dust.

Sensitisation to antigenic components in the dust occurred in some office workers and led to pyrexial illness on Mondays. The mechanism of the in-vivo pyrogen granule release is not known, but certainly antigen-antibody complexes can cause pyrogen granule release from polymorphonuclear leucocytes ${ }^{\mathbf{1 6}}$ ascan lymphokines derived from lymphocyte-antigen interactions. ${ }^{17}$ When tested about $44 \%$ of the factory workforce also had precipitins against humidifier sludge in the absence of disease. Thus it seems that either the office workers are sensitised differently from the factory workers or the presentation of the antigenic components are different in the office compared with the factory. Indeed, both factors may be operating but the latter is favoured since it is unlikely all the sensitised factory workers had a different type of sensitisation compared with the office workers, and the episodes were experienced only by people in the office.

The determination of undefined protozoa as antigen sources ${ }^{12}$ led to our obtaining a named free-living water-borne amoeba to prepare extracts. $N$ gruberi provided a suitable antigen source but other amoebae have since been shown to be antigen sources. Warhurst ${ }^{18}$ showed antibodies to Acanthamoeba in the sera of humidifier fever cases, and this is important in view of the limited pathogenic role of strains of Acanthamoeba. ${ }^{19}$

Amoebae and protozoa probably provide the common thread running through outbreaks of humidifier fever. Several workers have reported a multitude of organisms in humidifiers, ${ }^{15}$ and the existence of variations of the sewage-fungus complex seems likely. Our cross-reactions with the Spanish 
and Swedish sera indicate a distribution of similar antigens in humidifier fever, yet this differs from the report by Longbottom, ${ }^{20}$ who found only minor antigens to be common in outbreaks at three different locations in Britain. Also other organisms can mediate a pyrexial response-for example, Gram-negative organisms in sewage sludge disease. ${ }^{21}$

As in other organic dust diseases removal from exposure eradicates the disease, and this was so in our cases when housed in the temporary offices. A permanent solution to the problem for the office workers has been to construct a separate, detached office block to high environmental standards. Further remedial action has eliminated dust in the old office area, and the humidifier now contains less that $1 \%$ sludge compared with before cleaning. Yet within the factory the airborne dust still retains its potential to develop antigen when incubated with water for 10-14 days, and it seems logical that the microbes responsible were derived from the factory atmosphere since incoming rayon bales incubated with water did not develop antigen. The stringent cleaning routines operating, however, and the awareness of the factory management of the problem makes the build-up of antigenic material to an unacceptable level extremely unlikely.

Clinical investigations were undertaken by $\operatorname{Dr} \mathrm{A}$ Axford; airborne dust collections by $\mathrm{Mr} \mathrm{J}$ W Skidmore; pulmonary function testing by Mrs C Bevan and Mrs I Ashton; chest radiographs by Mr W Audsley-all of the MRC Pneumoconiosis Unit, Penarth; fungal analyses by Dr J Mullins, Asthma Research, Sully; and initial protozoal investigations by $\mathrm{Dr} A$ Griffiths, Microbiology, Cardiff. Vital help was provided by Drs A Jones and M Jones, Employment Medical Advisory Service, Cardiff. The kind participation of the employees at the factory under study, particularly $\mathrm{Mr} \mathrm{J}$ Tonge, Mr C Verbeeck, Mrs M Dew, Mr P Harbord, Mr R Stone is greatly appreciated as is the help and guidance of Dr J C Gilson, MRC Pneumoconiosis Unit. Finally, the artistic and photographic expertise of Mr D Llewellyn and Mr R Boothby, Llandough, is self-evident.

\section{References}

${ }^{1}$ Pickering CAC, Moore WKS, Lacey J, Holford-Strevens VC, Pepys $J$. Investigation of a respiratory disease associated with an air-conditioning system. Clin Allergy' $1976 ; 6: 109-18$.

${ }^{2}$ Pestalozzi C. Febrile Gruppenerkrankungen in einer. Modellschreinerei durch Inhalation von mit Schimmelpilzen kontaminiertem Befeuchterwasser (Befeuchtfieber). Schweiz Med Wochenschr 1959;89:710-3.

${ }^{3}$ Banaszak EF, Thiede WH, Fink JN. Hypersensitivity pneumonitis due to contamination of an air conditioner. $N$ Eng $J$ Med 1970;283:271-6.

${ }^{4}$ Sweet LC, Anderson JA, Callies QC, Coates EO, jun. Hypersensitivity pneumonitis related to a home furnace humidifier. J Allergy Clin Immunol 1971;48:171-8.

${ }^{5}$ Keller H, Spengler H, Latscha U. Befeuchterfieber. Schweiz Med Wochenschr 1972;102:865-72.

- Edwards JH, Baker JT, Davies BH. Precipitin test negative farmer's lung - activation of the alternative pathway of complement by mouldy hay dusts. Clin Allergy 1974;4: 379-88.

${ }^{7}$ Morgan DC, Smyth JT, Lister RW, Pethybridge RJ. Chest symptoms and farmer's lung: a community survey. Brit $J$ Ind Med 1973;30:259-65.

${ }^{8}$ Andersen AA. New sampler for the collection, sizing and enumeration of viable airborne particles. $J$ Bacteriol 1958;76:471-84.

${ }^{9}$ Cotes JE. Lung function. Assessment and application in medicine. 2nd ed. Oxford: Blackwell, 1968:388.

${ }^{10}$ Edwards $\mathrm{JH}$. The double dialysis method of producing farmer's lung antigens. $J$ Lab Clin Med 1972;79:683-8.

11 Working Party of the British Society for Mycopathology. In: Evans EGV, ed. Serology of Fungal Infection and Farmer's Lung Disease. Leeds: University Printing Service, 1976:9.

12 Edwards JH, Griffiths AJ, Mullins J. Protozoa as sources of antigen in 'humidifier fever'. Nature 1976;264:438-9.

${ }^{13}$ Dubois M, Gilles KA, Hamilton JK, Rebers PA, Smith F. Colorimetric method for determination of sugars and related substances. Anal Chem 1956;28:350-9.

${ }^{14}$ Lacey J, Lacey ME. Spore concentrations in the air of farm buildings. Trans Brit Mycolog Soc 1964;47:547-52.

${ }^{15}$ Curtis EJC. Sewage fungus, its nature and effects. Water Res 1969;3:289-93.

${ }^{16}$ Cranston WI. Humidifier fever. Mechanisms of pyrogenic responses. Thorax 1977;32:653-63.

${ }^{17}$ Chao P, Francis L, Atkins E. The release of an endogenous pyrogen from guinea pig leukocytes in vitro. $J$ Exp Med $1977 ; 145: 1288-98$.

${ }^{18}$ Warhurst D. Humidifier fever. Amoebae and allergic lung disease. Thorax 1977;32:653-63.

${ }^{19}$ Stamm WP. Humidifier fever. Pathogenicity of free living amoebae. Thorax 1977;32:653-63.

${ }^{20}$ Longbottom JL. Humidifier fever. Serological testing in humidifier fever. Thorax 1977;32:653-63.

${ }^{21}$ Rylander CR. Humidified fever. Responses to airborne sludge. Thorax 1977;32:653-63. 